Case Report

\title{
MULTIPLE VARIATIONS OF BRANCHES OF ABDOM INAL AORTA : A CASE STUDY
}

\author{
Shivarama C.H. ${ }^{1}$, Bhat Shivarama ${ }^{2}$, Shetty R.K. ${ }^{3}$, Avadhani R. ${ }^{4}$ \\ ${ }^{1} \mathrm{PG} / \mathrm{Tutor},{ }^{2}$ Associate Professor, ${ }^{3}$ Assistant Professor, ${ }^{4}$ Professor, \\ Department of Anatomy, Yenepoya M edical College, Mangalore - 575 018, India. \\ Correspondence: \\ Ramakrishna Avadhani, \\ M obile : 9845253560 E-mail : rkavadhani@ rediffmail.com
}

\begin{abstract}
:
M ultiple variations of the branches of abdominal aorta were observed during a routine dissection of the abdominal region in a 66-yearold male cadaver in the Department of Anatomy, Yenepoya M edical College, Yenepoya University, Mangalore. In the present case, both the inferior phrenic arteries arise from the celiac trunk. Left gastric artery is originates directly from abdominal aorta higher then celiac trunk. An accessory hepatic artery arises from the superior mesenteric artery. An accessory left renal artery found originating from the abdominal aorta. Right testicular artery arises as anterior branch of abdominal aorta. Knowledge of these variations could help surgeons to identify and protect the branches of abdominal aorta during surgery.
\end{abstract}

Keywords: Abdominal aorta, celiactrunk, hepatic artery, phrenic artery, renal artery, variations.

\section{Introduction:}

The abdominal aorta (AA) begins at aortic hiatus of the diaphragm, anterior to the inferior border of the 12th thoracic vertebra. It descends anterior to the lumbar vertebrae to end at the lower border of the 4th lumbar vertebra, by dividing into two common iliac arteries. The branches of AA are grouped into anterior, lateral and dorsal branches. The celiac trunk (CT) is the first anterior branch of $A A$ and it arises from the AA immediately below the aortic hiatus at the level of T12-L1 vertebra. The left gastric, splenic and common hepatic arteries all arise from the coeliac trunk ${ }^{1}$. Superior mesenteric artery (SM A) originates $1 \mathrm{~cm}$ below the $\mathrm{CT}$, at the level of the $\mathrm{L} 1-\mathrm{L} 2$ intervertebral disc. The inferior mesenteric artery (IMA) arises from the anterior or left anterolateral aspect of the AA at the level of L3 and 3-4 cm above aortic bifurcation. Inferior phrenic arteries (IPA) arise just above the $\mathrm{CT}$. They arise separately from the anterior aspect of the AA, just above the coeliac trunk ${ }^{1}$. The left and right renal arteries (RA) originate just below SMA and the right arises slightly higher than the left ${ }^{1}$. Gonadal arteries (GA) arise inferior to the RA. Variations in $A A$ and its branches are frequently observed and they occur due to embryological developmental changes.

Knowledge of morphology of $A A$ and its branches is important in regards to renal transplantation, renal trauma surgery, radiological imaging and surgical treatment of aortic aneurysms. Variations of the branches of AA and their relations to surrounding structures are important in regards to intra-abdominal surgery. The arteries that show frequent variations include $\mathrm{CT}, \mathrm{RA}$ and $\mathrm{GA}$. Ligation or damage of these arteries without knowing the possible variations in laparotomy, nephrectomy, renal transplantation, arterial reconstruction and laparoscopy or in other surgical applications may cause unpredictable complications, such as segmental or total visceral ischemia and failure. Although these variations might encounter in different cadavers, it is rare to find all these variations in the same cadaver.

\section{Materials and Methods:}

During the dissection of abdomen for the $1^{\text {st }}$ year MBBS students we found the multiple variations in the branching pattern of abdominal aorta. After the completion of the dissection we removed the abdominal aorta along with the branches and mounted it by using wires. Photographs are taken along with viscera and after mounting.

\section{Observations:}

The following variations were observed

1. The inferior phrenic arteries arise from the celiac trunk. 
2. The left gastric artery arises from the abdominal aorta just above the coeliac trunk.

3. Superior mesenteric gives rise to accessory hepatic artery.

4. One accessory renal artery is observed on the left side.

5. Right testicular artery arises from the anterior part of abdominal aorta.

\section{Discussion:}

Variations in the branches of the abdominal aorta were observed during a routine abdominal region dissection of a

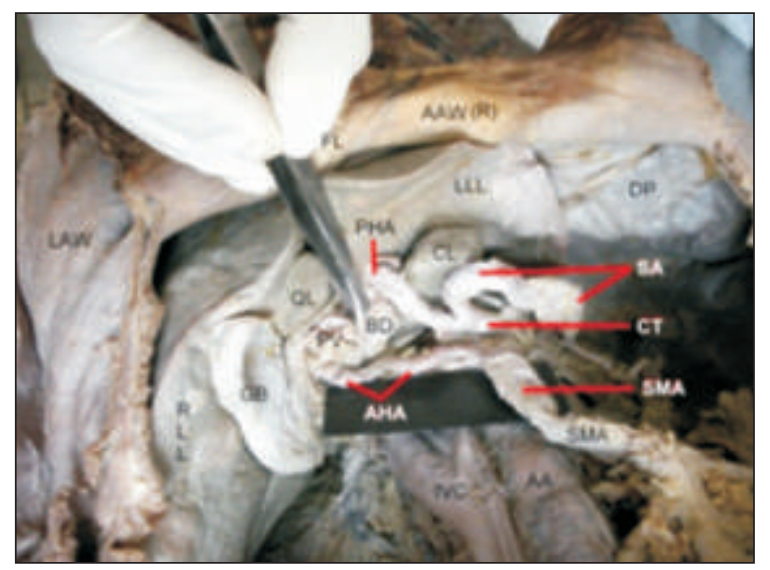

Fig.1 Accessory hepatic artery (AHA) from superior mesenteric artery (SM A) AAW (R)-Anterior abdominal wall (reflected), LAWLateral abdominal wall FL-Falciform ligament, RLL-Right lobe of liver,LLL-Leftlobe of liver GB-Gall bladder, QL-Quadrate lobe, CLCaudate lobe, DP-Diaphragm IVC-Inferior vena cava, AAAbdominal aorta,PV- Portal vein,BD-Bile duct CT-Coeliac trunk, PHA-Proper hepatic artery, SA-Splenic artery SMA-Superior mesenteric artery,AHA-Accessory hepatic artery from SM A.

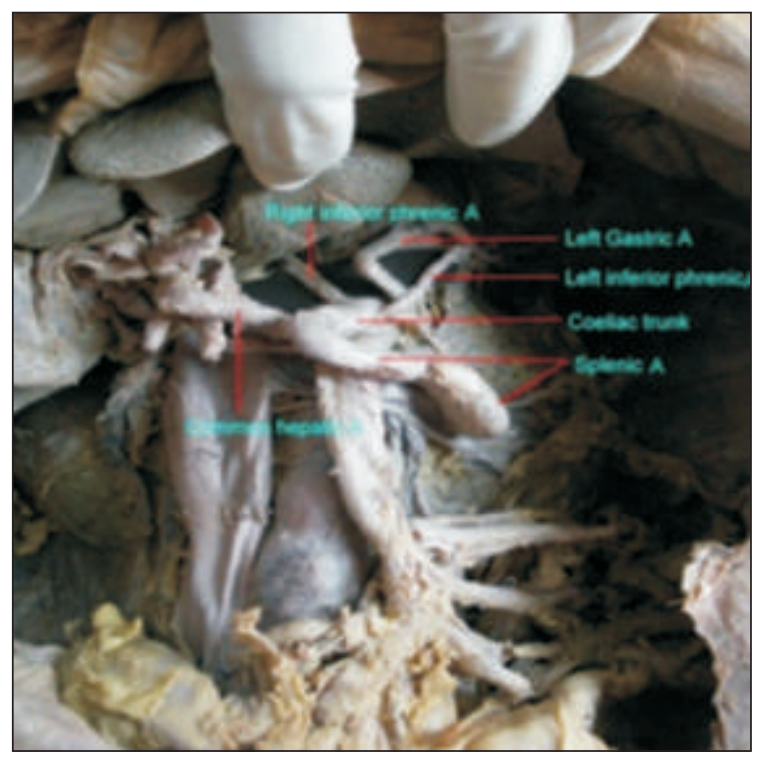

Fig.2 Branching of coeliac trunk. 66-year-old male cadaver in the Department of Anatomy, Yenepoya M edical College, M angalore. The inferior phrenic

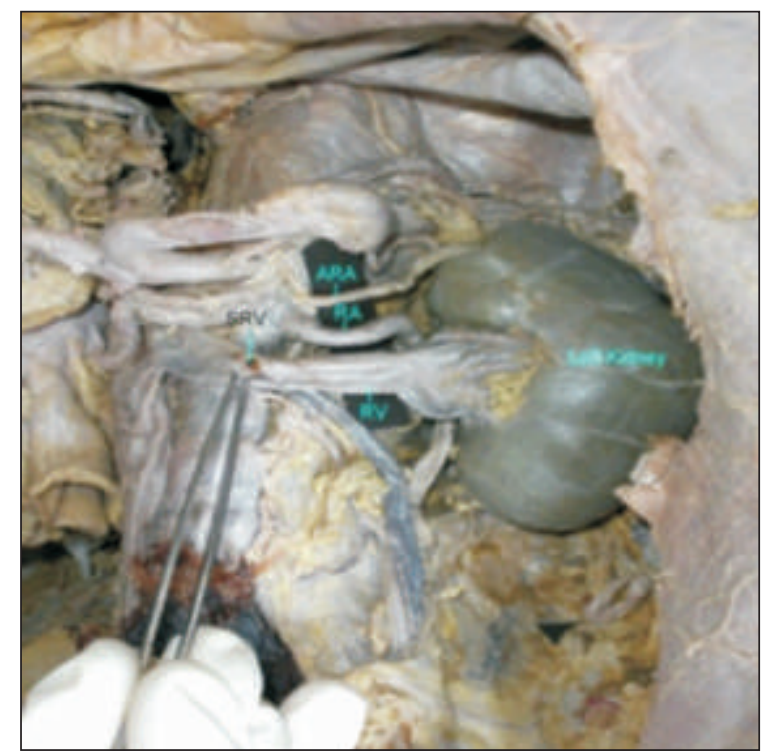

Fig.3 Left renal artery (RA) and accessory renal artery (ARA). RVLeft renal vein, SRV-Left suprarenal vein.

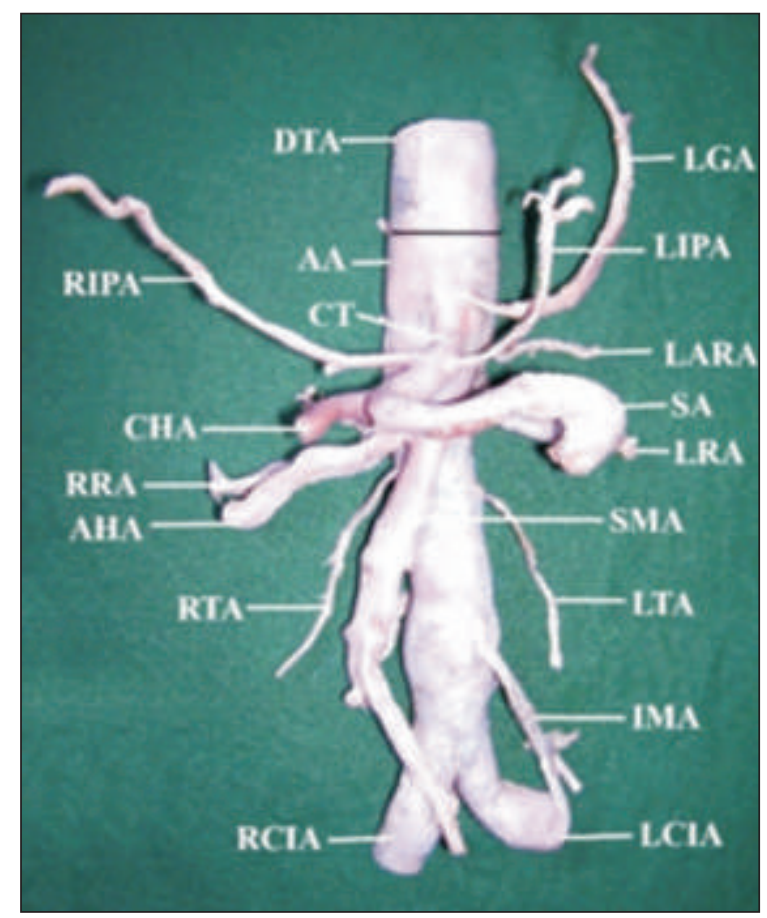

Fig.4 Different arteries arising from the abdominal aorta (Black line demarkabdominal aorta from descending thoracic aorta). DTA-Descending thoracic aorta, AA-Abdominal aorta,LGA-Left gastric artery,CT-Coeliac trunk, CHA-Common hepatic artery, SASplenic artery, RIPA-Right inferior phrenic artery, LIPA- Left inferior phrenic artery, LTA-Left testicular artery, RTA-Right testicular artery,SM A-Superior mesenteric artery,LRA-Left renal artery, LARA- Left accessory renal artery,AHA- Accessory hepatic artery from SMA,IMA- Inferior mesenteric artery, LCIA-Left common iliac artery, RCIA-Right common iliac artery. 
arteries arise from the celiac trunk. The left gastric artery arises from the abdominal aorta just above the coeliac trunk. The common hepatic artery and splenic artery arises from the coeliac trunk itself. Superior mesenteric artery arises from abdominal aorta but it gives a branch to the liver. It is accessory hepatic artery which supplies to the right lobe of the liver (Figs. 1, $2 \& 4$ ). Two renal arteries are seen on the left side. The upper artery evaluated as accessory renal artery to upper pole of left kidney, lower artery as the left renal artery (Figs.3\&4).Right testicular artery arises from anterior part of abdominal aorta but left testicular artery is arising from lateral part of abdominal aorta in the normal level.

Apart from normal branching of the coeliac trunk, many more branching variations have been reported in the literature ${ }^{4,5,6}$. Coeliac trunk may also give off one or both inferior phrenic arteries ${ }^{8}$. Loukas et al studied the origin of the left and right inferior phrenic arteries as a single rooted artery or separately, and determined that it originated from the coeliac trunk or the aorta as a single root ${ }^{2}$. The left gastric artery is the initial branch of the coeliac trunk. Previous studies have reported that the left gastric artery

\section{References :}

1. Moore KL, Dalley AF. Clinically Oriented Anatomy.In: Kelly PJ. $5^{\text {th }}$ Edition, Lippincott, Williams \& Wilkins, Philadelphia, 2005, pp. 253327.

2. Loukas M , Hullett J, Wagner T. Clinical anatomy of the inferior phrenic artery. Clinical Anatomy2005; 18:357-65.

3. Yildirim M, Ozan $\mathrm{H}$, Kutoglu T., Left gastric artery originating directly from the aorta. Surgical and Radiological Anatomy, 1998; 20:303-5.

4. Nayak S. Common celiaco - mesenterico - phrenic trunk and renal vascular variations., Saudi M edical Journal 2006; 27:1894-6. parted directly from the abdominal aorta ${ }^{3}$.The superior mesenteric artery may be the source of the common hepatic, gastroduodenal, accessory right hepatic, accessory pancreatic, or splenic arteries. ${ }^{8}$.ne or two accessory renal arteries frequently occur, especially on the left, usually from the aorta above or below the main artery, the former slightly more often ${ }^{8}$.

\section{Conclusion :}

The inferior phrenic artery, coeliac trunk, left gastric artery, superior mesenteric artery and renal artery variations were seen in our case, unlike in previously reported cases with single variations from the abdominal aorta. Hence, :many observed variations and extensions can result in unnoticed hemorrhage as a result of cutting of the vessel, or ischemia caused by the ligature of a vessel during surgery. We believe that awareness of these variations is important for surgeons who are interested in this field, so as to be able to detect and protect these arteries during surgery.

\section{Acknowledgements:}

The authors are grateful to Yenepoya University for permission to carry out this study.

5. Deepthinath R, Satheesha Nayak B, Mehta RB, et al. Multiple variations in the paired arteries of the abdominal aorta. Clinical Anatomy 2006; 19:566-8.

6. Vandamme JP, Bonte J. The branches of the celiac trunk., Acta Anatomica (Basel) 1985;122:110-4.

7. Uysal I I, Cicekcibasi A E, Yilmaz M T, Seker M, Sanli O .Multiple variations of the abdominal aorta in a single cadaver, Singapore Medical Journal 2010; 51(5):94-7.

8. Williams PL. et al. Gray's Anatomy in: Giorgio Gabella.38 ${ }^{\text {th }}$ Edition. Churchill Livingstone, 1995, pp. 1548-57. 